\title{
Comparison of the in Vitro and in Vivo Release of Digoxin from Four Different Soft Gelatin Capsule Formulations
}

\author{
J. G. Wagner, ${ }^{1,5}$ R. G. Stoll, ${ }^{2}$ D. J. Weidler, ${ }^{3}$ J. W. Ayres, ${ }^{1,4}$ \\ M. R. Hallmark, ${ }^{1}$ E. Sakmar, ${ }^{1}$ and A. Yacobi ${ }^{2}$
}

Received August 10, 1978-Final October 17, 1978

\begin{abstract}
A blinded, four-treatment crossover study in 16 normal adult male volunteers compared plasma concentrations and urinary excretion of digoxin, measured by radioimmunoassay, after oral administration of soft gelatin capsule formulations of digoxin. Four $0.4-m g$ formulations with different in vitro "burst times" and dissolution rates were administered, with 2-week intervals between treatments. The two capsules with lowest in vitro burst times (2.9 and $16 \mathrm{~min})$ gave comparable in vivo results. The other two capsules, with in vitro burst times of 62 and $229 \mathrm{~min}$, produced significant delays in digoxin absorption. In vitro-in vivo correlations were obtained by comparing the logarithm of the in vitro burst time with time to peak plasma level and the time to the first measurable plasma level $(\geq 0.05 \mathrm{ng} / \mathrm{ml})$. Also, the mean time to peak plasma level correlated with the logarithm of the time required to release either $50 \%$ or $85 \%$ of the digoxin in vitro. No significant changes were found in the amount of digoxin absorbed from each capsule as determined by urinary excretion or $A U C_{0-\infty}$.
\end{abstract}

KEY WORDS: digoxin bioavailability; in vitro-in vivo correlations; digoxin radioimmunoassay; plasma concentrations and urinary excretion of digoxin; gelatin capsule formulations.

This work was supported by Arnar-Stone Laboratories, Inc., McGraw Park, Illinois.

${ }^{1}$ College of Pharmacy and Upjohn Center for Clinical Pharmacology, The University of Michigan Medical Center, Ann Arbor, Michigan 48109.

${ }^{2}$ Department of Biopharmaceutical Sciences, Annar-Stone Laboratories, Inc., McGraw Park, Illinois 60085 .

${ }^{3}$ Department of Internal Medicine and Upjohn Center for Clinical Pharmacology, The University of Michigan Medical Center, Ann Arbor, Michigan 48109.

${ }^{4}$ Present address: School of Pharmacy, Oregon State University, Corvallis, Oregon 97331.

${ }^{5}$ Address all correspondence to J. G. W. 


\section{INTRODUCTION}

Previous investigators (1-5) have reported that soft gelatin capsules containing digoxin in solution have enhanced bioavailability associated with reduced between-subject variability in digoxin plasma concentrations compared with digoxin tablets. Stoll et al. (6) reported that digoxin soft gelatin capsules, prepared by Arnar-Stone, Inc., showed considerably less within-subject variability than digoxin tablets when both capsules and tablets were administered twice to each subject of the panel. It should be noted that treatments $B_{1}$ and $B_{2}$ in the study of Stoll et al. (6) employed the same digoxin soft gelatin capsule as treatment $\mathrm{A}$ in the study reported in this article. The purpose of this investigation was to establish in vitro quality control limits for the digoxin soft elastic gelatin capsules by evaluating the in vivo absorption of capsules with varying in vitro release rates.

\section{EXPERIMENTAL}

\section{Human Study}

Sixteen adult male volunteers with no known disease who weighed between 61 and $92 \mathrm{~kg}$ and were between 21 and 34 years of age were selected. Before each subject could be included in the study, a complete physical examination, routine blood analysis and urinalysis, and electrocardiogram were carried out. As a screening procedure, values for the tests were required to be in the normal range. Informed consent was obtained from each subject.

A recent drug history was taken for each prospective subject. All subjects participating in the study received no barbituates or other enzymeinducing agents for a period of 30 days preceding initiation of the study and none concurrent with it. They received no other medication or alcoholic beverages for a period of 7 days before initiation of the study and none during the study.

At 9 p.m. each night before dosing with digoxin, the subjects ate a late snack. From 10 p.m. the night before dosing to $4 \mathrm{hr}$ after dosing, the subjects fasted. On the days of dosing they ate standard lunches and dinners which were not high in fat or protein content. Subjects drank lemonade and received no tea or coffee on the day of dosing or throughout the period of blood and urine collection.

The human study was performed "double blind." Arnar-Stone Laboratories, Inc., sent to The University of Michigan four bottles of capsules simply labeled A, B, C, and D. These were administered to the subjects as indicated by the study plan shown in Table I. The first day of one 
phase was separated by another phase by a period of 14 days. Not until all plasma and urine samples had been analyzed and the results obtained and recorded were the investigators who performed the human study informed of the "burst times" and the dissolution $t_{50 \%}$ and $t_{85 \%}$ values of the four lots of digoxin capsules designated by A, B, C, and D in Table I.

Fifteen milliliters of whole blood was taken at zero time (just before dosing) and $7 \mathrm{ml}$ was taken at $0.25,0.5,0.75,1,1.5,2,3,4,6,8,12,24,48$, 72 , and $96 \mathrm{hr}$ after drug administration. Blood was drawn in vacutainers containing dried $\mathrm{Na}_{2}$ EDTA. Plasma, obtained by centrifugation shortly after withdrawal of the blood, was quick-frozen and stored at $-20^{\circ} \mathrm{C}$ until just before assay. Urine was collected in the intervals -2 to $0,0-24,24-48$, and $48-72 \mathrm{hr}$. After measurement of the volume and adequate mixing, a $30-\mathrm{ml}$ aliquot of each urine was frozen and maintained at $-20^{\circ} \mathrm{C}$ until just prior to assay.

\section{Assay of Plasma and Urine Samples}

Plasma and urine samples were assayed by the radioimmunoassay method of Wagner et al. (7). This assay measures digoxin down to $0.05 \mathrm{ng}$ digoxin per milliliter of plasma. As described (7), the normalized fraction of digoxin bound, $F$, is given by

$$
F=B(x) / B(0)
$$

and data were fitted to the biexponential equation shown as

$$
F=P(1) e^{-P(2) C}+P(3) e^{-P(4) C}
$$

In equation $1, B(x)$ is the percent digoxin bound at the concentration $C$ and $B(0)$ is the percent digoxin bound in the absence of digoxin. In equation $2, C$ is the concentration of digoxin in $\mathrm{ng} / \mathrm{ml}$ and the $P(i)$ 's $(i=1-4)$ are parameters estimated by digital computer fitting. When calibration data were plotted and fitted in this study, concentrations were the ordinate values (rather than the abscissa values as before) and $F$ values were the abscissa

Table I. Treatment Schedule in Human Study

\begin{tabular}{cccccc}
\hline & & \multicolumn{3}{c}{ Treatment $\begin{array}{c}\text { in indicated } \\
\text { phase }\end{array}$} \\
\cline { 3 - 7 } Group & Subjects in group & I & II & III & IV \\
\hline 1 & $2,5,9,14$ & A & B & D & C \\
2 & $4,8,10,13$ & B & C & A & D \\
3 & $1,6,11,16$ & C & D & B & A \\
4 & $3,7,12,15$ & D & A & C & B \\
\hline
\end{tabular}


values (rather than the ordinate values as in ref. 7). Thus each set of calibration data was fitted to the biexponential equation

$$
C=P(1) e^{-P(2) F}+P(3) e^{-P(4) F}
$$

with reciprocal weighting of the concentrations. An example of such a plot is shown in Fig. 1. The advantage of equation 3 is that once $F$ is available for an "unknown" plasma sample and the $P(i)$ 's are available from fitting the calibration data via digital computer, the value of $C$ for the unknown is calculated directly. When using the former fitting method and equation 2 , the concentration, $C$, of each unknown had to be estimated by an iterative method which took much more time. Very low plasma concentrations of digoxin were estimated by a different method than indicated by equation 3 . The "line value" of $F$, corresponding to $C=0.1$, which is 0.834 in the example shown in Fig. 1, was found by an iterative procedure. A linear equation was then established by joining the points $C=0.1, \hat{F}=0.834$ and $C=0, F=1.0$ thus the equation for estimating digoxin concentrations where $F>0.834$ in the example is

$$
C=0.6024(1-F)
$$

since 0.6024 is both the intercept and the slope of such a line. The values of $\hat{F}$ corresponding to $C=0.1$ varied with the particular calibration data, hence equation 4 varied withe particular calibration data. The average of the two independent assays was used as the digoxin concentration in each determination.

A set of 60 unknown plasma samples (15 samples/treatment $\times 4$ treatments) and five samples for calibration purposes (corresponding to $C=0.1,0.5,1,2$, and $5 \mathrm{ng}$ digoxin $/ \mathrm{ml}$ plasma) was assayed independently by each of the two analysts in a given day. The 64 zero-hour plasmas (16 subjects $\times 4$ treatments/subject) were assayed by each analyst in 1 day, and they were treated as unknowns. The mean apparent average digoxin concentrations were zero $(\overline{<} 0.05 \mathrm{ng} / \mathrm{ml})$ after each treatment, which conformed with the expected zero values of those samples if there was no carryover.

\section{In Vitro Tests}

The in vitro release tests were carried out similar to a dissolution test by a U.S.P. rotating basket apparatus (8) using a dissolution medium consisting of $500 \mathrm{ml}$ of distilled water and $3 \mathrm{ml}$ of concentrated hydrochloric acid. The basket was rotated at $120 \pm 5 \mathrm{rmp}$ and the fluid was maintained at $37^{\circ} \mathrm{C}$. One capsule was placed in the basket for each test. The dissolution test was repeated six times for each lot of capsules. Serial samples were taken at specified times. An equal volume of dilute hydrochloric acid was added after 


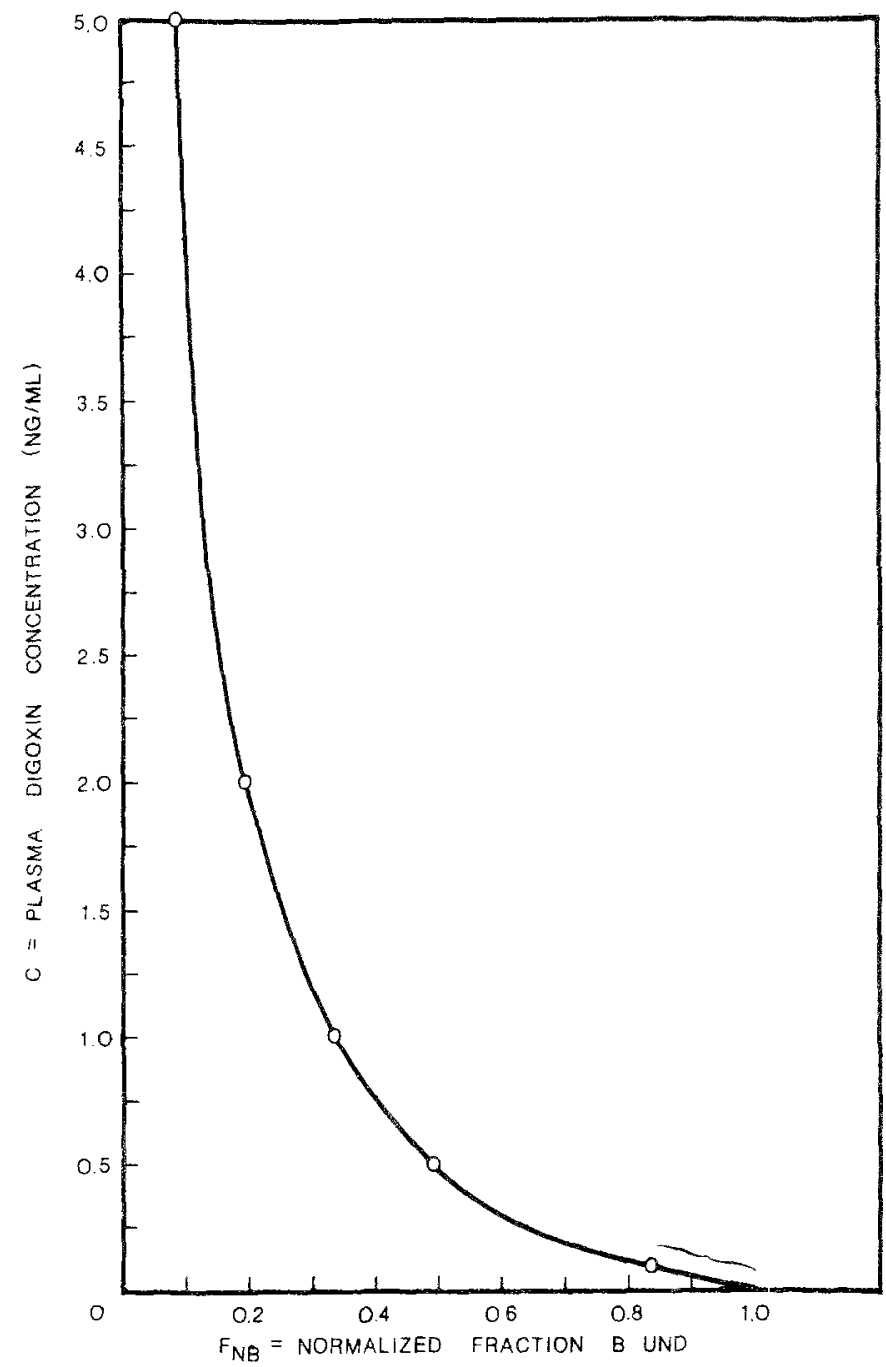

Fig. 1. Typical calibration curve for digoxin radioimmunoassay. Part corresponding to plasma concentrations of $0.1,0.5,1,2$, and $5 \mathrm{gg} / \mathrm{ml}$ where $F \leq 0.834$ was fitted by the equation $C(\mathrm{ng} / \mathrm{ml})=$ $4.841 e^{-4.648 F}+60.751 e^{-38.828 F}$. Bracketed part where $1.0 \geq F \geq 0.834$ was fitted by equation 4 .

withdrawal of each sample. The digoxin concentration in each sample was determined by a fluorometric procedure (9). "Burst time" is defined as the time required for rupture of the tested capsule. The times for $50 \%$ and $85 \%$ of the digoxin to be released ( $t_{50 \%}$ and $t_{85 \%}$, respectively) were determined graphically after plotting milligrams of digoxin in solution vs. time. 


\section{Evaluation of Results}

Plasma concentrations at each sampling time, peak plasma concentrations, times of the peak plasma concentrations, areas under the plasma concentration curves, and other pharmacokinetic parameters were analyzed by analysis of variance (10a). Tukey's allowable difference was calculated (10b).

$\mathrm{AUC}_{0-\infty}$ for each subject after each treatment was calculated by the classical method using

$$
\mathrm{AUC}_{0-\infty}=\mathrm{AUC}_{0-96}+\hat{C}_{96} / \beta
$$

and also by fitting an orthogonal least-squares line through points of a plot of $(\mathrm{AUC})_{i}$ vs. $(\mathrm{AUC})_{i+1}-(\mathrm{AUC})_{i}$ as indicated by

$$
(\mathrm{AUC})_{i}=\mathrm{AUC}_{0-\infty}-\left[\frac{1}{1-e^{-\overline{\beta \Delta t}}}\right]\left[(\mathrm{AUC})_{i+1}-(\mathrm{AUC})_{i}\right]
$$

These are methods III and IIC as described by Wagner and Ayres (11). These methods utilize only the values of $\mathrm{AUC}_{0-24}, \mathrm{AUC}_{0-48}, \mathrm{AUC}_{0-72}$, and $\mathrm{AUC}_{0-96}$ obtained with the trapezoidal rule (10c) for each case (11). In equation $5, \mathrm{AUC}_{0-96}$ is the trapezoidal area from zero to $96 \mathrm{hr}, \hat{C}_{96}$ is the "line value" of the plasma concentration at $96 \mathrm{hr}$, and $\beta$ is the apparent elimination rate constant when the $\log$-linear $C_{p}, t$ data were fitted to

$$
\ln C_{p}=\ln B-\beta t
$$

by the method of least squares (i.e., $\ln C_{p}$ vs. $t$ ). In equation $6, \Delta t$ is the constant time interval, namely $24 \mathrm{hr}$.

Relative efficiencies of absorption $\left(F_{\mathrm{B}} / F_{\mathrm{A}}, F_{\mathrm{C}} / F_{\mathrm{A}}\right.$, and $\left.F_{\mathrm{D}} / F_{\mathrm{A}}\right)$ of digoxin were estimated from individual subject data by four different methods as follows, where $x$ represents treatment $\mathrm{B}, \mathrm{C}$, or D and $\mathrm{A}$ represents treatment $\mathrm{A}$.

\section{Method 1}

Method 1 assumes that the plasma clearance of digoxin is constant and is based on

$$
F_{x} / F_{\mathrm{A}}=D_{\mathrm{A}}(\mathrm{AUC})_{x} / D_{x}(\mathrm{AUC})_{\mathrm{A}}
$$

In equation 8 the symbol $D$ refers to the oral dose.

\section{Method 2}

Method 2 is the nonrigorous method of Kwan and Till (12) and assumes that the nonrenal component of plasma clearance remains constant, but a 
correction is made for changing renal clearance. The method is based on

$$
F_{x} / F_{\mathrm{A}}=D_{\mathrm{A}}(\mathrm{AUC})_{x} / D_{x}(\mathrm{AUC})_{\mathrm{A}}-\left[\mathrm{Cl}_{r}^{\mathrm{A}}-\mathrm{Cl}_{r}^{x}\right](\mathrm{AUC})_{x} / D_{x}
$$

where $\mathrm{Cl}_{r}$ refers to renal clearance of digoxin.

\section{Method 3}

Method 3, based on

$$
F_{x} / F_{\mathrm{A}}=\left(D_{\mathrm{A}}\right)\left(\mathrm{Cl}_{r}^{x}\right)(\mathrm{AUC})_{x} /\left(D_{x}\right)\left(\mathrm{Cl}_{r}^{\mathrm{A}}\right)(\mathrm{AUC})_{\mathrm{A}}
$$

assumes that any change in renal clearance of digoxin is accompanied by a proportional change in nonrenal clearance.

\section{Method 4}

Method 4, based on the dose-corrected amounts excreted in the urine, utilizes

$$
F_{x} / F_{\mathrm{A}}=\left(A_{e}\right)_{x}\left(D_{\mathrm{A}}\right) /\left(A_{e}\right)_{\mathrm{A}}\left(D_{x}\right)
$$

where $A_{e}$ refers to apparent amount of digoxin excreted in the urine in $72 \mathrm{hr}$ according to radioimmunoassay. Although mathematically methods 3 and 4 are equivalent, the values obtained by use of equations 10 and 11 will be different since equation 10 utilizes AUC data while equation 11 utilizes $A e$ data.

Renal clearance of digoxin for each subject after each treatment was estimated as the slope of orthogonal least-squares line (9) when $A e$ was plotted vs. AUC. Three points were used in each case, corresponding to $0-24,24-48$, and $48-72 \mathrm{hr}$.

\section{RESULTS AND EVALUATION}

Table II summarizes the mean digoxin plasma concentrations, the amounts excreted in the urine in $72 \mathrm{hr}$, pharmacokinetic parameters, and results of statistical analyses. If any two of the mean plasma concentrations differ by more than the value of Tukey's allowable difference, then the difference in means is significant $(p \leq 0.05)$. The differences in means for treatments $A$ and $C$ were not significant at any sampling time. Similarly, the means of the other parameters listed in Table II for treatments $A$ and $C$ were strikingly similar and the differences in means were not significant.

Figure 2 is a plot of mean plasma concentrations for only the first $6 \mathrm{hr}$, which discloses the differences in the rate of rise of plasma concentrations, and, by inference, differences in absorption rate of digoxin. The order was $\mathrm{A}=\mathrm{C}>\mathrm{B}>\mathrm{D}$. 


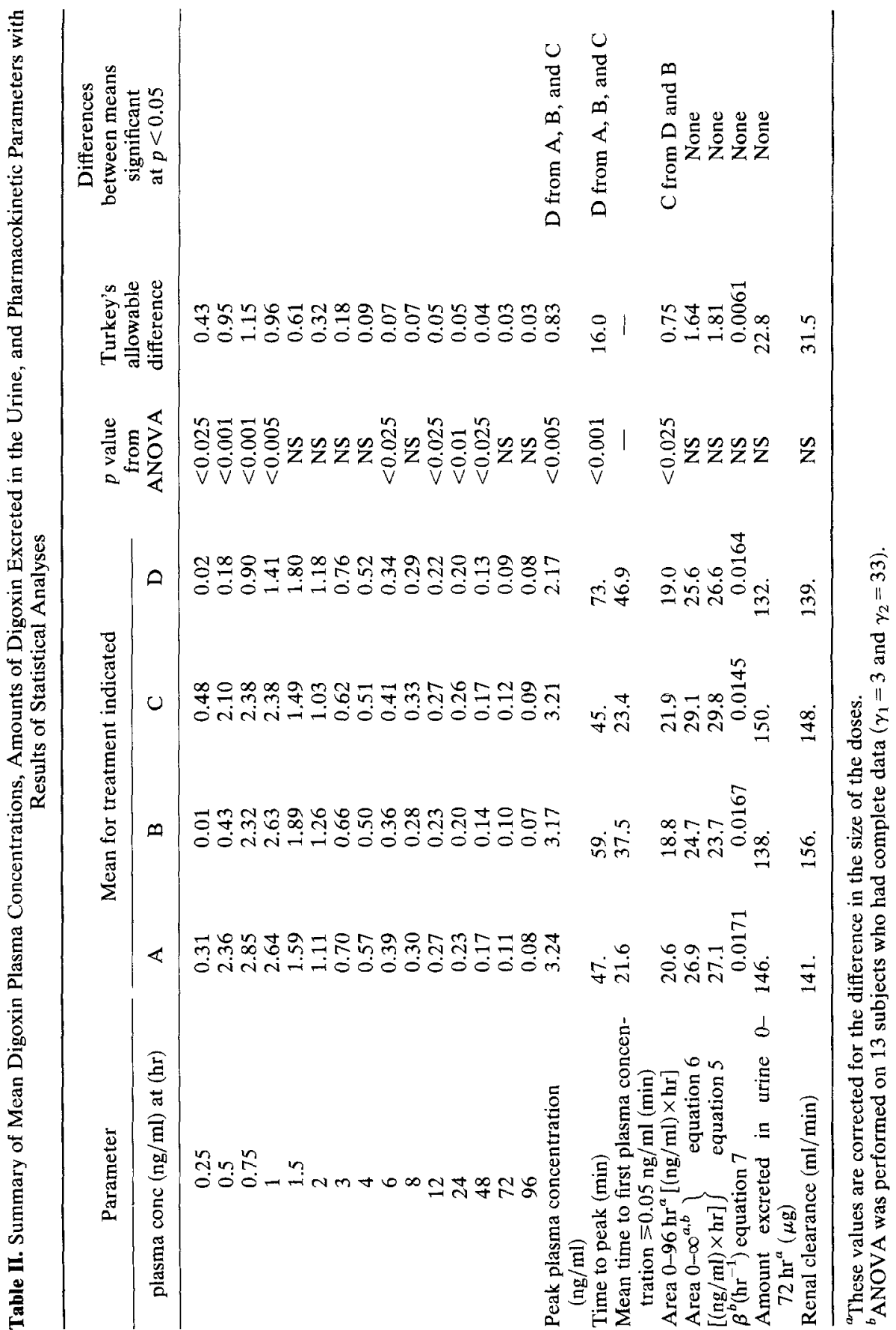




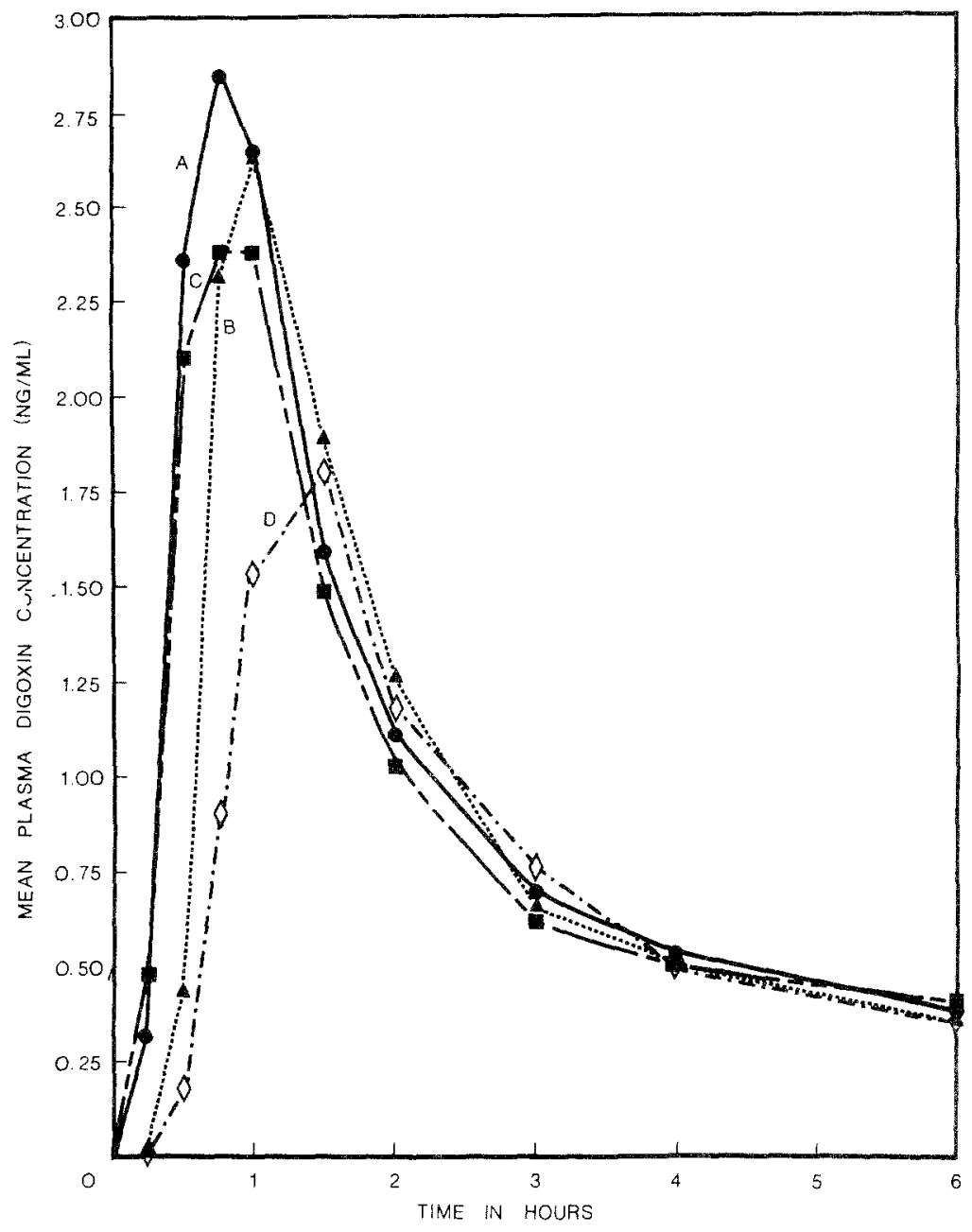

Fig. 2. Mean plasma digoxin concentrations during first $6 \mathrm{hr}$ after administration. Letters indicate treatments.

The relative absorption efficiencies of digoxin estimated four different ways are listed in Table III. If the average absorption efficiencies are equal, one expects the mean ratio not to differ significantly from unity. The significance of this difference was tested by $t$ test. In all cases, the mean ratio was not significantly different from unity $(p>0.10$ or $p>0.25)$. Thus, although the capsules used for treatments $B$ and $D$ gave different absorption rates of digoxin than treatments $A$ and $C$, all four capsules provided very similar efficiencies of absorption of digoxin. 
Table III. Relative Absorption Efficiencies of Digoxin Estimated Four Different Ways

\begin{tabular}{lcrlcccc}
\hline & & & & \multicolumn{3}{c}{ Student } \\
Ratio & Method & Equation & Mean & Range & C.V. $(\%)$ & $t^{a}$ & $p$ value \\
\hline$F_{\mathrm{B}} / F_{\mathrm{A}}$ & 1 & 7 & 0.989 & $0.533-1.61$ & 34.7 & 0.12 & $>0.25$ \\
& 2 & 8 & 1.06 & $0.456-1.74$ & 39.2 & 0.56 & $>0.25$ \\
& 3 & 9 & 1.22 & $0.397-2.26$ & 47.0 & 1.49 & $>0.10$ \\
& 4 & 10 & 0.983 & $0.550-1.94$ & 32.2 & 0.21 & $>0.25$ \\
$F_{\mathrm{C}} / F_{\mathrm{A}}$ & 1 & 7 & 1.19 & $0.741-2.68$ & 40.9 & 1.51 & $>0.10$ \\
& 2 & 8 & 1.22 & $0.738-2.88$ & 43.7 & 1.59 & $>0.10$ \\
& 3 & 9 & 1.33 & $0.537-3.64$ & 56.5 & 1.70 & $>0.10$ \\
$F_{\mathrm{D}} / F_{\mathrm{A}}$ & 4 & 10 & 1.08 & $0.515-2.14$ & 35.6 & 0.83 & $>0.25$ \\
& 1 & 7 & 1.00 & $0.470-1.38$ & 28.9 & 0.00 & $>0.25$ \\
& 2 & 8 & 0.988 & $0.504-1.46$ & 31.9 & 0.14 & $>0.25$ \\
& 3 & 9 & 1.11 & $0.548-2.15$ & 52.8 & 0.68 & $>0.25$ \\
& 4 & 10 & 0.974 & $0.438-2.26$ & 43.9 & 0.24 & $>0.25$ \\
\hline
\end{tabular}

${ }^{a} t$ value for testing the significance of difference of the mean value from unity.

Table IV. In Vitro Characteristics of Digoxin Capsules

\begin{tabular}{lcccc}
\hline & \multicolumn{5}{c}{ Treatment } \\
\cline { 2 - 5 } & $\mathrm{A}$ & $\mathrm{B}$ & $\mathrm{C}$ & $\mathrm{D}$ \\
\hline Mean potency (mg/cap) & 0.4078 & 0.3974 & 0.3974 & 0.3650 \\
SD of mean potency (mg/cap) & 0.0024 & 0.0027 & 0.0027 & 0.0016 \\
Burst time (min) & 2.9 & 62. & 16. & 229. \\
$t_{50 \%}(\min )$ & $<10$. & 60. & 30. & 150. \\
$t_{85 \%}(\min )$ & $<10$. & 95. & 50. & 180. \\
\hline
\end{tabular}

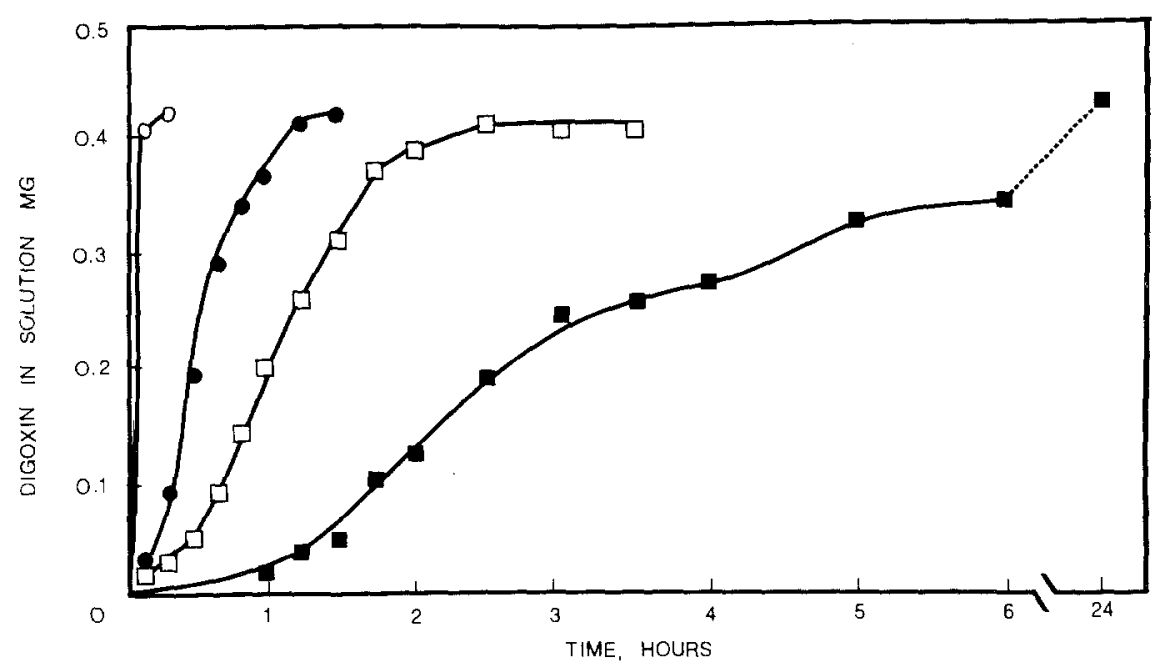

Fig. 3. Dissolution profiles of the four lots of digoxin capsules. Each point represents average amount released from six capsules. Key: $O$, treatment $A ;$, treatment $C ; \square$, treatment $B ; \mathbf{E}$, treatment $\mathrm{D}$. 


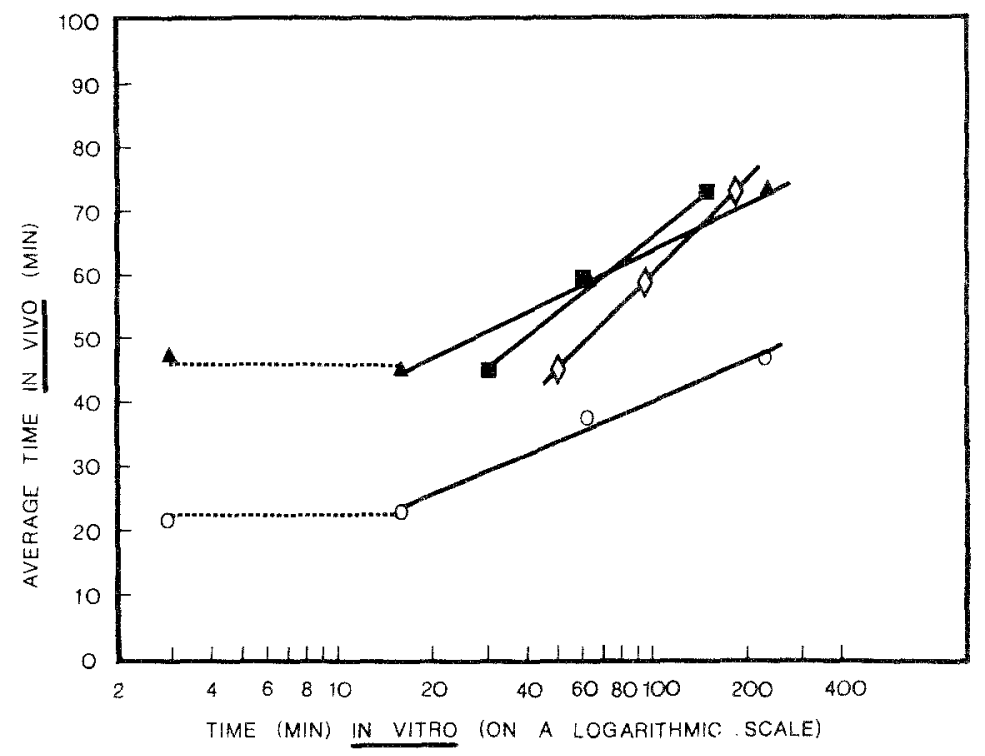

Fig. 4. In vitro-in vivo correlations.

\section{KEY}

\begin{tabular}{|c|c|}
\hline Code & Abscissa $(x)$ \\
\hline $\boldsymbol{\Delta}$ & $\log$ (burst time) \\
\hline & $\log \left(t_{50 \%}\right)$ \\
\hline$\diamond$ & $\log \left(t_{85 \%}\right)$ \\
\hline 0 & $\log$ (burst time) \\
\hline
\end{tabular}

Ordinate $(y)$

mean time to peak piasma level mean time to peak plasma level mean time to peak plasma level mean time to plasma level $\geq 0.05 \mathrm{ng} / \mathrm{ml}$

The in vitro characteristics of the capsules are listed in Table IV, and the release profiles are shown in Fig. 3. The mean potencies listed in Table IV were used as the doses in applying equations 8-11.

Figure 4 depicts four different in vitro-in vivo correlations involving treatments $\mathrm{B}, \mathrm{C}$, and $\mathrm{D}$. Since results in vivo were not significantly different following treatments $\mathrm{A}$ and $\mathrm{C}$, treatment $\mathrm{A}$ was not included in the correlations. This finding is in accordance with the dissolution theory that there will be a range of in vitro dissolution rates where the in vivo parameters do not change (13). This is the case with the digoxin capsules used as treatments $\mathrm{A}$ and $\mathrm{C}$.

\section{REFERENCES}

1. G. I. Mallis, D. H. Schmidt, and J. Lindenbaum. Superior bioavailability of digoxin solution in capsules. Clin. Pharmacol. Ther. 18:761-768 (1975).

2. B. F. Johnson, C. Bye, G. Jones, and G. A. Sabey. A completely absorbed oral preparation of digoxin. Clin. Pharmacol. Ther. 19:746-751 (1976). 
3. B. F. Johnson, G. Smith, and J. French. The comparability of dosage regimens of Lanoxin tablets and Lanoxicaps. Br. J. Clin. Pharmacol. 4:209-211 (1977).

4. P. Ghirardi, G. Catenazzo, O. Mantoro, G. C. Merotti, and A. Marzo. Bioavailability of digoxin in a new soluble pharmaceutical formulation in capsules. J. Pharm. Sci. 66:267269 (1977).

5. J. Lindenbaum. Greater biovailability of digoxin solution in capsules: Studies in the postprandial state. Clin. Pharmacol. Ther. 21:278-282 (1977).

6. R. G. Stoll, R. A. Schwartz, G. C. Chao, A. Yacobi, D. J. Weidler, J. W. Ayres, E. Sakmar, M. R. Hallmark, and J. G. Wagner. The assessment of the intrasubject variability in digoxin absorption in man after administration of two oral dosage forms. Clin. Pharmacol. Ther. 23:131 (1978) (abst.).

7. J. G. Wagner, M. R. Hallmark, E. Sakmar, and J. W. Ayres. Sensitive radioimmunoassay for digoxin in plasma and urine. Steroids 29:787-807 (1977).

8. U.S. Pharmacopeia XVIII, 1970, pp, 934-935.

9. D. Wells, B. Katzung, and F. H. Myers. Spectrofluorometric analysis of cardiotonic steroids. J. Pharm. Pharmacol. 13:389-395 (1961).

10. J. G. Wagner. Fundamentals of Clinical Pharmacokinetics, Drug Intelligence Publications, Hamilton, Ill., 1975, (a) pp. 290-297; (b) p. 300; (c) p. 71.

11. J. G. Wagner and J. W. Ayres. Bioavailability assessment: methods to estimate total area $\left(\mathrm{AUC}_{0-\infty}\right)$ and total amount excreted $\left(A_{e}^{\infty}\right)$ and importance of blood and urine sampling scheme with application to digoxin. J. Pharmacokin. Biopharm. 5:533 -557 (1977).

12. K. C. Kwan and A. E. Till. Novel method for biovailability assessment. J. Pharm. Sci. 62:1494-1497 (1973).

13. J. G. Wagner. Drug bioavailability studies. Hosp. Pract. 12:119-127 (1977). 